propuesta de regionalización. Arch Cardiol Mex. 2010;80: 133-40.

2. San Luis-Miranda R, Arias-Monroy LG, Peralta-Pedrero ML, et al. Guía de práctica clínica: persistencia del conducto arterioso. Rev Med Inst Mex Seguro Soc. 2012;50:453-63.

3. Ruiz-González MD, Gómez-Guzmán E, Párraga-Quiles MJ, et al. Ductus arterioso persistente [Internet]. España: Protocolos Diagnóstico Terapéuticos AEP; 2008 [consultado Oct 2015]. Disponible en: https://www.aeped.es/sites/default/files/ documentos/36.pdf

4. Golombek S, Sola A, Baquero H, et al. Primer Consenso clínico del SIBEN: enfoque diagnóstico y terapéutico del ductus arterioso permeable en recién nacidos pre término. An Pediatr (Barc). 2008;69:454-81.

5. Ying-Yao C, Hsiao-Ping W, Jenn-Tzong C, et al. Perinatal factors in patent ductus arteriosus in very low-birth weight infants. Pediatr Int. 2014;56:72-6.

6. Tapia-Rombo C, González Arenas M, Carpio-Hernández JC, et al. Un índice del diámetro interno del conducto arterioso/superficie corporal como necesidad de cierre del conducto en el recién nacido de pretérmino. Rev Invest Clin. 2013;65:12-23.

7. Simon SR, van Zogchel L, Bas-Suárez MP, et al. Platelet counts and patent ductus arteriosus in preterm infants: A systematic review and meta-analysis. Neonatology. 2015;108:143-51.

8. Tapia-Rombo CA, Calvo-Rangel KM, Saucedo-Zavala VJ, et al. Evolución final de recién nacidos prematuros operados y no operados por persistencia del conducto arterioso. Gac Med Mex. 2008;144:111-20.
9. Pacifici G. Clinical pharmacology of Furosemide in neonates: A review. Pharmaceuticals. 2013;6:1094-129.

Ana Margarita Martínez-Roque ${ }^{\mathrm{a}}$, Jaime Valle Leal ${ }^{\mathrm{a}, *}$, Alba J. Martínez Limón ${ }^{\mathrm{b}}$ y Lucia Álvarez-Bastidas ${ }^{\mathrm{c}}$

a Servicio de Pediatría, Hospital General Regional Numero Uno, IMSS, Ciudad Obregón, Sonora, México

b Departamento de Neonatología, Hospital General Regional Numero Uno, IMSS, Ciudad Obregón, Sonora, México

' Departamento de Enseñanza e Investigación, Hospital General Regional Número Uno, IMSS, Ciudad Obregón, Sonora, México

* Autor para correspondencia. Guerrero y Huisaguay s/n, Col. Bellavista, Ciudad Obregón, Sonora, México.

Teléfono: +52 6441270367 .

Correo electrónico: valle_jaime1@hotmail.com

(J. Valle Leal).

http://dx.doi.org/10.1016/j.acmx.2016.05.008

1405-9940/

(c) 2016 Instituto Nacional de Cardiología Ignacio Chávez. Publicado por Masson Doyma México S.A. Este es un artículo Open Access bajo la CC BY-NC-ND licencia (http: / / creativecommons.org/ licencias/by-nc-nd/4.0/).

\section{Prevalencia de factores de riesgo de erosión en pacientes con cierre percutáneo de comunicación interauricular ${ }^{\text {th }}$}

\section{Prevalence of risk factors of erosion in patients with percutaneous closure of atrial septal defects}

El cierre percutáneo de comunicación interauricular (CIA) tipo ostium secundum ha demostrado tener una tasa de éxito similar al tratamiento quirúrgico, menor tiempo de internación y una tasa de mortalidad idéntica ${ }^{1}$. Sin embargo, no está exento de complicaciones, y una de las más temidas es la erosión de estructuras cardiacas adyacentes al dispositivo ${ }^{2}$. La incidencia de erosiones no es completamente conocida y solo existen estimaciones. Según reportes de la base de datos MAUDE, en diferentes momentos de seguimiento, la incidencia varía entre $0.1-0.3 \%$ de la población tratada con tasas de mortalidad entre el $20-30 \%^{3-5}$. Múltiples factores de riesgo han sido identificados, siendo la ausencia o deficiencia del borde aórtico el mencionado con mayor frecuencia ${ }^{6}$. El objetivo del presente trabajo fue reportar la prevalencia

\footnotetext{
Este trabajo formó parte del Trabajo Final del Máster de Ecocardiografía Transesofágica, Universidad Internacional Menéndez Pelayo. Sociedad Española de Cardiología. Asociación de Ecocardiografía e Imágenes Cardiovasculares de la Sociedad Interamericana de Cardiología.
}

de factores de riesgo de erosión en una serie de pacientes consecutivos tratados con cierre percutáneo de CIA.

\section{Métodos}

Realizamos un análisis retrospectivo que incluyó a todos los pacientes referidos para cierre percutáneo de $\mathrm{CIA}$ entre noviembre de 2013 a mayo de 2015. Los pacientes fueron intervenidos bajo anestesia general y monitorizados durante el procedimiento con ecocardiografía transesofágica bidimensional (GE Vivid ${ }^{\circledR}$ S5, Sonda 6T-RC). Se revisaron las historias clínicas incluyendo los datos hemodinámicos del procedimiento y las imágenes del ETE intraprocedimiento. Los bordes del defecto fueron evaluados en vistas esofágicas medias-bajas en 3 diferentes planos $\left(0,45\right.$ y $\left.90^{\circ}\right)$. El borde anterosuperior o retroaórtico fue medido en planos intermedios $\left(30-50^{\circ}\right)$ y fue definido como deficiente cuando su tamaño fue menor a $5 \mathrm{~mm}$, o ausente cuando no se observaba. La deficiencia o ausencia del resto de los bordes fueron definidas con el mismo criterio.

Se definieron factores de riesgo de erosión según la bibliografía $a^{3}:$ 1) al menos un borde deficiente o ausente; 2) CIA grande (mayor a $30 \mathrm{~mm}$ o relación defecto/peso mayor a 1.5); 3) septum interauricular mal alineado; 4) movilidad exagerada del borde posterior; 5) cabalgamiento del dispositivo sobre la raíz aórtica, y 6) sobredimensionamiento, tamaño del dispositivo/diámetro del defecto mayor a 1.5. Las variables categóricas se expresan en porcentaje, y las variables continuas en promedio y rango. Para el análisis estadístico se utilizó el paquete InfoStat versión profesional (Universidad Nacional de Córdoba, 2014). 

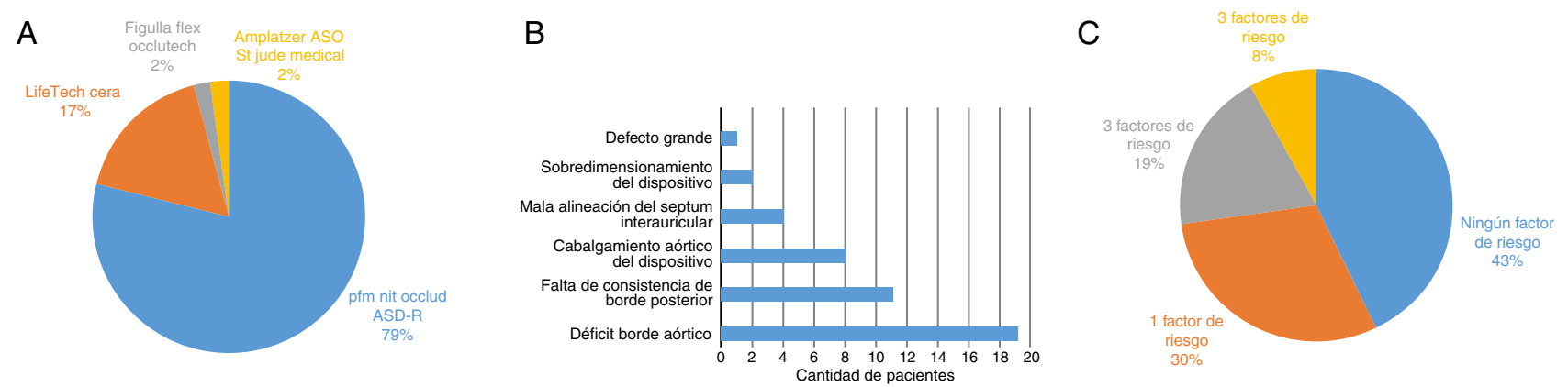

Figura 1 A) Dispositivos implantados. B) Prevalencia de factores de riesgo de erosión. C) Número de factores de riesgo por paciente.

\begin{tabular}{ll} 
Tabla 1 & \multicolumn{2}{l}{ Características generales } \\
\hline Edad & 17.8 años $(4-60 \mathrm{años})$ \\
Tamaño CIA & $16 \mathrm{~mm}(7-35 \mathrm{~mm})$ \\
$\begin{array}{l}\text { Tamaño CIA con balón (stop } \\
\text { flow) }\end{array}$ & $19.9 \mathrm{~mm}(12-35 \mathrm{~mm})$ \\
$\begin{array}{l}\text { Relación tamaño CIA/peso } \\
\text { Tamaño de dispositivo }\end{array}$ & $0.51(0.10-1.03)$ \\
$\begin{array}{l}\text { Relación tamaño } \\
\text { dispositivo/tamaño CIA }\end{array}$ & $1.28(1.11-2.00)$ \\
$\begin{array}{l}\text { Presión sistólica de arteria } \\
\text { pulmonar }\end{array}$ & $33 \mathrm{mmHg}(18-57 \mathrm{mmHg})$ \\
$\begin{array}{l}\text { Tiempo de radioscopia } \\
\text { CIA: comunicación interauricular. }\end{array}$ & $8.7 \mathrm{~min}(2.5-20 \mathrm{~min})$ \\
\hline
\end{tabular}

\section{Resultados}

Se incluyeron 47 pacientes con CIA tipo ostium secundum, 37 de ellos mujeres (79\%). Durante el procedimiento se realizó la técnica de «stop-flow» con balón elastomérico en 38 pacientes $(81 \%)$. El cierre percutáneo fue exitoso en el $100 \%$ de los casos (tabla 1). El dispositivo usado con mayor frecuencia fue Nit-Occlud ${ }^{\circledR}$ PFM (fig. 1A). Con respecto a los factores de riesgo de erosión, en el $40.4 \%$ (19 pacientes) se objetivó un borde retroaórtico deficiente, no hubo deficiencias de otros bordes y ningún paciente tuvo ausencias de alguno de los bordes. El 23.4\% (11 pacientes) mostró falta de consistencia del borde posterior, el 8.5\% (4 pacientes) tuvo mala alineación del septum interauricular con respecto a la raíz aórtica, en el $2.1 \%$ (un paciente) se observó un defecto de tamaño grande $(35 \mathrm{~mm})$, en el $17 \%$ ( 8 pacientes) el dispositivo fue colocado cabalgando la raíz aórtica, y hubo sobredimensionamiento en 2 pacientes (4.2\%) (fig. $1 \mathrm{~B})$. De los 47 pacientes incluidos, 20 pacientes $(43 \%)$ no presentaron ningún factor de riesgo, los restantes evidenciaron 1,2 o 3 factores de riesgo $(30,19$ y $8 \%$, respectivamente) (fig. 1C). Todos los pacientes tuvieron un ecocardiograma transtorácico de control a las $24 \mathrm{~h}$ del procedimiento, y no se observó presencia de derrame pericárdico en ningún caso.

\section{Discusión}

Las 2 características más frecuentemente encontradas en los casos publicados de erosión incluyen: la deficiencia del borde anterosuperior o retroaórtico, que se ha reportado en el $90 \%$ de los casos de erosión y el sobredimensionamiento del dispositivo con respecto al tamaño del defecto ${ }^{3}$. La fisiopatología de la erosión no ha sido claramente dilucidada, y por lo tanto, los factores de riesgo identificados no explican la totalidad de los casos de erosión reportados ${ }^{7}$. Las características de riesgo propuestas, aumentan la chance de contacto entre el dispositivo y el techo auricular. Se sugiere que la erosión está relacionada a un mecanismo abrasivo entre el dispositivo y el tejido vecino, posiblemente magnificado por el mayor contacto (sobredimensionamiento, defecto de gran tamaño, cabalgamiento aórtico) y el movimiento «en sierra» del dispositivo (borde posterior móvil, protrusión del dispositivo sobre la aorta $)^{6}$. El potencial abrasivo podría estar relacionado además, a la composición de los dispositivos y la susceptibilidad del paciente, ya que la infrecuente incidencia de erosión no se correlaciona con la frecuencia de ausencia o deficiencia del borde aórtico.

En nuestra experiencia, observamos que, la prevalencia de factores de riesgo de erosión es muy frecuente, siendo la deficiencia de borde aórtico el más comúnmente encontrado. Esto coincide con reportes previos en donde el déficit del borde aórtico se encuentra entre el $40-50 \%$ de los $\operatorname{casos}^{8,9}$. En conclusión, es frecuente el cierre de la $\mathrm{CIA}$ en presencia de características anatómicas de riesgo de erosión, por lo que estos pacientes deberían tener seguimiento estricto más prolongado, ya que existen casos publicados de erosiones hasta 8 años después de implantado el dispositivo ${ }^{10}$.

\section{Financiación}

No se recibió patrocinio de ningún tipo para llevar a cabo este artículo.

\section{Bibliografía}

1. Butera G, Biondi-Zoccai G, Sangiorgi G, et al. Percutaneous versus surgical closure of secundum atrial septal defects: A systematic review and meta-analysis of currently available clinical evidence. Eurolntervention. 2011;3:377-85.

2. Moore J, Hegde S, El-Said H, et al. Transcatheter device closure of atrial septal defects. A safety review. J Am Coll Cardiol Cardiovasc Interv. 2013;6:433-42.

3. Amin Z, Hijazi ZM, Bass JL, et al. Erosion of Amplatzer septal occluder device after closure of secundum atrial septal 
defects: Review of registry of complications and recommendatios to minimize future risk. Catheter Cardiovasc Interv. 2004;63:496-502.

4. DiBardino DJ, McElhinney DF, Kaza AK, et al. Analysis of the US Food and Drug Administration manufacturer and user facility device experience database for adverse events involving Amplatzer Septal Occluder devices and comparison with the Society of Thoracic Surgery Congenital Cardiac Surgery database. J Thorac Cardiovasc Surg. 2009;137:1334-41.

5. Crawford GB, Brindis RG, Krucoff MW, et al. Percutaneous atrial septal occluder devices and cardiac erosion: A review of the literature. Catheter Cardiovasc Interv. 2012;80:157-67.

6. Amin Z. Echocardiographic predictors of cardiac erosion after Amplatzer septal occluder placement. Catheter Cardiovasc Interv. 2014;83:84-92.

7. Diab K, Kenny D, Hijazi ZM. Erosions, erosions, and erosions! Device closure of atrial septal defects: how safe is safe? Catheter Cardiovasc Interv. 2012;80:168-74.

8. Podnar T, Martanovic P, Gavora P, et al. Morphological variations of secundum-type atrial septal defects: Feasibility for percutaneous closure using Amplatzer septal occluders. Catheter Cardiovasc Interv. 2001;53:386-91.

9. OB́yrne ML, Glatz AC, Sunderji S, et al. Prevalence of deficient retro-aortic rim and its effects on outcomes in device closure of atrial septal defects. Pediatr Cardiol. 2014;35:1181-90.

10. Roberts WT, Parmar J, Rajathurai T. Very late erosion of Amplatzer septal occluder device presenting as pericardial pain and effusion 8 years after placement. Catheter Cardiovasc Interv. 2013;82:E592-4.

Alejandro E. Contreras ${ }^{a, *}$, Adolfo Ferrero Guadagnoli ${ }^{\mathrm{b}}$ y Alejandro Peirone ${ }^{b}$

a Servicio de Cardiología, Hospital Privado Universitario de Córdoba, Instituto Universitario de Ciencias Biomédicas de Córdoba, Córdoba, Argentina

b Servicio de Hemodinamia, Hospital Privado Universitario de Córdoba, Instituto Universitario de Ciencias Biomédicas de Córdoba, Córdoba, Argentina

* Autor para correspondencia. Naciones Unidas 346, CP X5016KEH, Barrio Parque Vélez Sársfield, Córdoba, Argentina; Teléfono: +543514688220; Fax: +433514688818.

Correo electrónico: aletreras@hotmail.com

(A.E. Contreras).

http://dx.doi.org/10.1016/j.acmx.2016.05.006 $1405-9940$

( 2016 Instituto Nacional de Cardiología Ignacio Chávez. Publicado por Masson Doyma México S.A. Este es un artículo Open Access bajo la CC BY-NC-ND licencia (http://creativecommons.org/ licencias/by-nc-nd/4.0/).

\section{Bloqueo auriculoventricular completo reversible durante el cierre percutáneo de comunicación interauricular}

\section{Transient complete atrioventricular block during percutaneous atrial septal defect closure}

El bloqueo completo del nódulo auriculoventricular (AV) posterior a un cierre percutáneo de comunicación interauricular tipo ostium secundum es una complicación infrecuente, y la mayoría de los casos se resuelven espontáneamente, siendo rara la necesidad de colocación de marcapasos.

\section{Informe de caso}

Presentamos el caso de un paciente femenino de 5 años de edad (14,3 kg de peso), con una comunicación interauricular tipo ostium secundum de $11 \mathrm{~mm}$ de diámetro mayor por ecocardiografía transtorácica bidimensional, bordes adecuados para cierre percutáneo, dilatación de cavidades cardíacas derechas e hipertensión arterial pulmonar leve (presión pulmonar media de $29 \mathrm{mmHg}$ ). El electrocardiograma muestra ritmo sinusal y conducción AV conservada. Se propone cierre percutáneo, el cual es realizado bajo control angiográfico y por ecocardiografía transtorácica. Durante el procedimiento, y previo a la elección del dispositivo oclusor, se realiza medición del defecto con balón elastomérico según técnica de «stop flow», el cual arroja $15 \mathrm{~mm}$ de diámetro, por lo cual se elige dispositivo NitOcclud $^{\circledR}$ ASD-R (PFM Medical, Alemania) de $16 \mathrm{~mm}$. Se coloca dispositivo sin complicaciones. Inmediatamente después de la apertura del disco derecho del dispositivo, y previa a la liberación del mismo, se observa en monitorización electrocardiográfica, bloqueo AV completo (fig. 1A), por lo que se decide recapturar el dispositivo y suspender el procedimiento. Después de esto, queda bajo control electrocardiográfico y se observa recuperación de la conducción AV hasta llegar a recuperar el ritmo sinusal 72 min después (figs. 1B-D). Se concluye el procedimiento y se decide esperar al crecimiento del niño para nuevo intento de cierre percutáneo.

Treinta y siete meses después del primer procedimiento, a los 7 años de edad ( $21 \mathrm{~kg}$ de peso), se intenta nuevamente el cierre percutáneo del defecto, bajo control ecocardiográfico transesofágico tridimensional (defecto de $12 \mathrm{~mm}$ en su diámetro mayor, fig. 2A). No se realiza medición con balón elastomérico y se intenta el cierre con el dispositivo NitOcclud $^{\circledR}$ ASD-R de $14 \mathrm{~mm}$ (fig. 2B). Se logra cerrar el defecto sin complicaciones y sin alteraciones en la conducción AV. Es dada de alta a las $24 \mathrm{~h}$, con ritmo sinusal y conducción AV conservada. Un nuevo control al mes del procedimiento muestra dispositivo implantado sin fuga residual y electrocardiograma con ritmo sinusal normal (fig. 2C).

\section{Discusión}

La presencia de bloqueo auriculoventricular durante o posterior al cierre percutáneo de una comunicación interauricular es poco frecuente, con una incidencia entre $0,23^{1}$ y $4,9 \%^{2}$. Un estudio retrospectivo que incluyo 610 pacientes, sometidos a cierre de foramen oval permeable y defectos del septum interauricular, demostró un incremento significativo de la duración de la onda $\mathrm{P}$ en electrocardiogramas 